\title{
CONTINUOUS FUNCTIONS OVER DISCRETE PARTIAL ORDERS
}

\author{
JOHN L. PFALTZ
}

\begin{abstract}
This paper examines the properties of structure preserving morphisms $f$ over discrete partial orders. It employs concepts of continuity and path homomorphisms. It will conclude that no single constraint on $f$ will be sufficient, and it will also conclude that a convexity constraint on $f^{-1}$ seems to be essential. We employ closure lattices to help reach this conclusion.
\end{abstract}

\section{INTRODUCTION}

Mathematics is hugely concerned with functions that preserve the essential structure of its mathematical objects even as they change specific details. We think of continuous functions, $f: R^{m} \rightarrow R^{n}$; of group and ring homomorphisms; and of automorphism concepts.

But, what constitutes a "structure preserving" morphism of a partially ordered set has not received as much study as these more familiar examples. This is surprising, because partial orders, or partially ordered sets, or posets, which are terms that we will use interchangeably, are common in applied mathematics. They are ubiquitous in computer science. They are readily a model of concurrent processing $[3,7]$, and any system of events occurring in time, such as task scheduling [8]. The theory of data types [9], and abstract models of computation, such as denotational semantics [33] have been extensively studied as "directed-complete partial orders", or dcpo's, in domain theory [1,16,37]. In "formal concept analysis", or FCA [15], the concepts have a partially ordered structure. There is no shortage of application instances.

In pure mathematics, lattice theory $[4,5,18]$ is the study of "well-behaved" partial orders; they are fundamental in some algebraic systems, such as "Stone Spaces" [21].

So, partial orders are widely used and very well understood - except for their functional change. Functions that map one partial order into another are far less easy to characterize.

\subsection{Basic terminology}

Partially ordered sets, denoted $(P, \leq)$, are a familiar mathematical system. The element ordering, $\leq$, is a pre-order if for all elements $x, y, z \in P$ we have

$M S C$ (2010): primary 06A06, 06A 11.

Keywords: partial order, transformation, homomorphism, convex subgraph inverse map. 


\begin{abstract}
(A1) $x \leq x$ If in addition

(A2) $x \leq y$ and $y \leq z$ imply that $x \leq z, \quad$ transitive

(A3) $x \leq y$ and $y \leq x$ imply that $x=y, \quad$ anti-symmetric $^{1}$

then $(P, \leq)$ is a partially ordered set. Usually these three axiomatic properties are presented together; but we shall see that (A3) is very different in character from (A2). While it is convenient to have $\leq$ be reflexive (A1); nothing essential is changed if it is omitted, or if the order is assumed to be "anti-reflexive" with $x \not \leq x$.
\end{abstract}

Let $(P, \leq)$ be a partially ordered set. An element $y$ is said to be a maximal element of $S \subseteq P$ if $y \in S$ and there exists no element $z \in S, z \neq y$ such that $y \leq z$. An element $y$ is said to be an upper bound, or $u . b$., of $S \subseteq P$ if for all $x \in S, x \leq y$. An element $y$ is said to be a least upper bound, l.u.b, of $S$ if it is an upper bound and if for all upper bounds $z_{i}$ of $S, y \leq z_{i}$. Minimal elements, lower bounds (l.b.) and greatest lower bounds (g.l.b.) are defined analogously.

A subset $S \subseteq P$ is said to be totally ordered if for all $x, y \in S, x \neq y$ implies either $x<y$ or $y<x$. A chain, $C(x, z) \subseteq P$ is a totally ordered subset $C$ with minimal element $x \in C$ and maximal element $z \in C$. A partial order $(P, \leq)$ is said to be discrete if for every chain $C(x, z)$, the set $C(x, z) \backslash\{z\}$ is a chain with a maximal element. (For this paper, we also assume that $C(x, z) \backslash\{x\}$ is a chain with a minimal element.) This distinguishes our approach to partial orders from that of domain theory $[1,16]$ which is largely concerned with convergence and approximation of least upper bounds in infinite partial orders.

Any finite partial order must be discrete. Discrete partial orders need not be finite.

Because we restrict ourselves to discrete partial orders, we can illustrate the concepts of this paper with directed graphs. A chain, $C(x, z)$ in $(P, \leq)$ can be visualized as a path $\rho(x, z)$ from $x$ to $z$, consisting of a sequence of elements $<x, \ldots, y_{i}, \ldots, z>$ where $x \leq y_{i} \leq z$. We shall use $\neg \rho$ to denote the non-existence of any path. Thus $x \neg \rho y$ is equivalent to $x \not \leq y$. By the length of $\rho(x, z)$, which we denote by $|\rho(x, z)|$, we mean the number of elements in the chain $C(x, z)$ less one. Thus, every element $y$ has a path $\rho(y, y)$ of length zero. If $y=\max (C(x, z) \backslash\{z\})$, we say $z$ covers $y$. Equivalently, we could say there is a "directed edge" between $y$ and $z .^{2}$ Figure 1 illustrates a partial order $\leq$ on $P=\{a, \ldots h\}$, in which $g$ covers $e$. We choose to represent partial orders in a horizontal (left to right) manner rather than the more conventional vertical Hasse diagram because it conserves space on the printed page. The arrow heads suggest the $\leq$ relation with "lesser" elements to the left. As with the $\leq$ relation, we will use $x \rho z$ to denote that there exists at least one path from $x$ to $z$, and let $\rho(x, z)$ denote a specific path, in much the same way that a chain denotes a specific total ordering. Those readers more comfortable with the order terminology using $\leq$ may replace every occurrence of $\rho$ with $\leq$.

\footnotetext{
${ }^{1}$ There are various names for (A3); some would call this "weak anti-symmetry" and want "anti-symmetry" to mean that $x<y$ implies $y \nless x$.

${ }^{2}$ It is customary to define graphs as an "edge" relation $E$ on the ground set of elements $P$, e.g., $[2,11,19,26]$. Since our focus is on transitive ordering relations it seems more natural to emphasize the "path" relation, and let edges denote covering situations.
} 


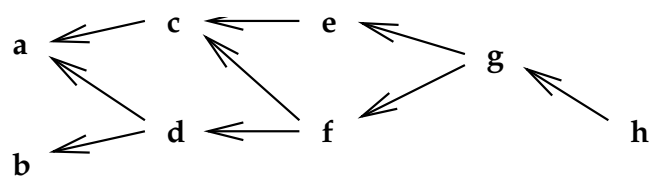

Figure 1. A representative partially ordered set $(P, \rho)$.

A cycle is a path $\rho\left(x, \ldots y_{i}, \ldots x\right)$ of length $\geq 2 .{ }^{3}$ A directed graph $(P, \rho)$ is said to be acyclic if it contains no cycles. It is well known that

Proposition 1.1. A discrete pre-ordered set $(P, \leq)$ is a partial order, if and only if its corresponding directed graph $(P, \rho)$ is acyclic.

\section{ORDER PRESERVING FUNCTIONS}

A function $f:(P, \leq) \rightarrow\left(P^{\prime}, \leq^{\prime}\right)$ is said to be order preserving, or isotone, if $x \leq z$ implies $f(x) \leq^{\prime} f(z)$. In our discrete case this is equivalent to saying $x \rho z$ implies $f(x) \rho^{\prime} f(z) .{ }^{4}$ However, order/path preserving functions need not preserve partial orders. This is most easily demonstrated when we visualize the partial orders as acyclic graphs. Let $(P, \rho)$ be that of Figure 1. Let $f(b)=f(h)=b^{\prime}=h^{\prime}$ and let $f(x)=x^{\prime}$ for $x=\{a, c, d, e, f, g\}$ as in Figure 2. While $f$ is readily path preserving,

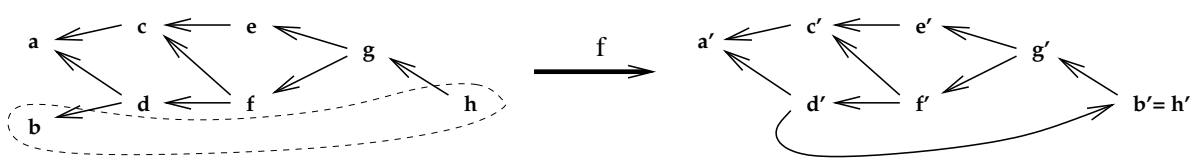

Figure 2. An order/path preserving function on Figure 1.

$\left(P^{\prime}, \rho^{\prime}\right)$ is not acyclic; it is not a partial order.

\subsection{Categorical posets}

In some texts $[10,31]$, the category, Poset, of partially ordered sets is defined to consist of the class of all partially ordered sets as its objects, and the class of all isotone functions $[A \stackrel{f}{\longrightarrow} B]$ between objects $A$ and $B$. It is easy to understand why this is often presented as an early example; it is not hard to show that the essential axioms of a category are satisfied, namely that the composition $f \cdot g$, of isotone functions $f$ and $g$, is isotone; that identity functions $i d_{A}$ and $i d_{B}$ exist for all objects $A, B$; and that $i d_{A} \cdot f$ and $f \cdot i d_{B}$ are isotone. But, does this category really characterize partial orders? It does characterize a preorder, which is any transitive order relation; but it does not capture the "antisymmetry" aspect. This is tacitly acknowledged in [1] where we have "If we drop antisymmetry from our list of requirement than we get what is known as preorders. This does not change

\footnotetext{
${ }^{3}$ In definition of "path" we assumed a path of length 0 at every node to assure reflexivity; consequently "loops" or edges of the form $(x, x)$ are simply ignored, if they exist.

${ }^{4}$ It is correct to denote the order/path relation on $P^{\prime}$ by $\leq^{\prime}$ and $\rho^{\prime}$, because the ordering relation may not be the same as on $P$.
} 
the theory very much." Mac Lane [24] never defines a poset category. The author knows of no definition of a Poset category that is distinct from that of a PreOrder or TotalOrder category; but see [36], which is quite relevant to this paper.

\subsection{Graph theoretic maps}

In graph theory, the term "homomorphism" is widely used to describe an "edge preserving" function $f: G \rightarrow G^{\prime}$ where $G=(P, E), G^{\prime}=\left(P^{\prime}, E^{\prime}\right)$ and $(x, y) \in$ $E$ implies $(f(x), f(y)) \in E^{\prime},[17,26]$. A function $f$ satisfying this definition of "homomorphism" is path preserving, or order preserving. Unfortunately, this common definition need not imply that $G$ and $G^{\prime}$ have similar structure. It is satisfied if $f(E) \subseteq E^{\prime}$. For example, every graph $G$ is the homomorphic image of the anti-chain (with $E=\emptyset$ ) on the same set $P$ of elements.

The notion of a "homomorphism" should imply that the essential "structure" of the graph is somehow preserved. Consequently, some authors prefer a stronger notion of "homomorphism". In this paper, we will call $f: G \rightarrow G^{\prime}$ a homomorphism if it is edge (order) preserving and $\left(x^{\prime}, y^{\prime}\right) \in E^{\prime}$ implies $\exists x, y \in P$ such that $(x, y) \in E, x^{\prime}=f(x), y^{\prime}=f(y)$.

However, even if $f: G \rightarrow G^{\prime}$ is a homomorphism in this stricter sense, and thus edge preserving, we can not assume $f:(P, \rho) \rightarrow\left(P^{\prime}, \rho^{\prime}\right)$ is a homomorphism with respect to $\rho$. Consider the function $f$ of Figure 3 in which $f\left(b_{1}\right)=f\left(b_{2}\right)=b^{\prime}$. It is a homomorphism of $(P, E)$ onto $\left(P^{\prime}, E^{\prime}\right)$, but not a homomorphism of $(P, \rho)$
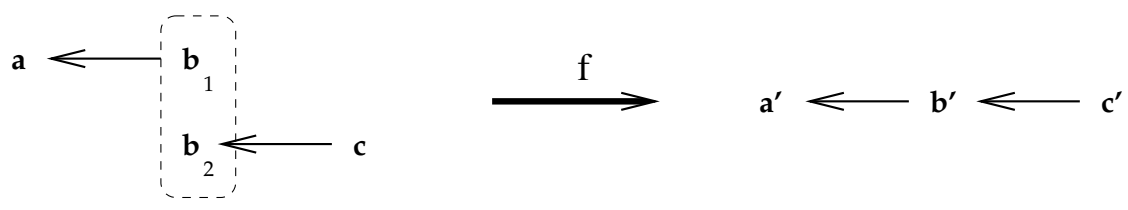

Figure 3. A homomorphism with respect to $E$ that is not homomorphic with respect to $\rho$.

because $\left(a^{\prime}, c^{\prime}\right) \in \rho^{\prime}$, although there is no $(a, c) \in \rho$. We can strengthen the homomorphism concept a bit more by requiring that if $x^{\prime} \rho^{\prime} z^{\prime}$ then $\exists x, z \in P$ such that $f(x)=x^{\prime}, f(z)=z^{\prime}$ and $x \rho z$. We call this a path homomorphism.

A subset $S \subseteq P$ is said to be convex if for all $x, z \in S, x \leq y \leq z$ implies $y \in S$. This terminology is more intuitive when we visualize the partial order as an acyclic graph. In this case, $x, z \in S$ and $x \rho y_{i} \rho z$ implies $y_{i} \in S$; or equivalently all paths between $x$ and $z$ lie within $S$, which is a traditional definition of convexity. In Figure 1, $S_{1}=\{a, c, d, f\}$ is convex; but $S_{2}=\{a, c, e, g\}$ is not, because $c \rho f \rho g$, but $f \notin S_{2}$. In particular, chains, or paths, need not be convex subsets. And cycles, such as $\left\{d^{\prime}, f^{\prime}, g^{\prime}, h^{\prime}\right\}$ in Figure 2, may be convex. Readily, if $(P, \rho)$ is acyclic, then every singleton subset $\{x\}$ is a convex subset, since any path of the form $x \rho y_{i} \rho x, y_{i} \neq x$, would constitute a cycle. In this paper, we will regard a path homomorphism $f$ as the standard for a "structure-preserving" map of a partially ordered set, provided it can be assured that $f(P)$ is acyclic. This latter constraint is non-trivial.

The following two propositions have been taken from [27], but their proofs are repeated here for the reader's convenience. 
Proposition 2.1. Let $f:(P, \rho) \rightarrow\left(P^{\prime}, \rho^{\prime}\right)$ be a homomorphism (of any kind). If $S^{\prime}$ is convex in $P^{\prime}$, then $S=f^{-1}\left(S^{\prime}\right)$ is convex in $P$.

Proof. Because $f$ is a homomorphism, $x \rho z$ in $P$ implies $x^{\prime} \rho^{\prime} z^{\prime}$ in $P^{\prime}$. If there were a path $x \rho y \rho z$ where $x, z \in f^{-1}\left(S^{\prime}\right)$, but $y \notin f^{-1}\left(S^{\prime}\right)$ then $y^{\prime}=f(y)$ would lie on a path between $x^{\prime}$ and $z^{\prime}$ in $S^{\prime}$ contradicting the convexity of $S^{\prime}$.

Thus, convexity is preserved under the inverse mappings of order preserving functions. But, $f(S)$ need not be convex, even if $S$ is. A homomorphism will be called a convex homomorphism if it takes convex subsets onto convex subsets. This definition is reminiscent of open functions in topology which take open sets onto open sets.

Proposition 2.2. Let $f:(P, \rho) \rightarrow\left(P^{\prime}, \rho^{\prime}\right)$ be a convex path homomorphism. If $(P, \rho)$ is acyclic, then $\left(P^{\prime}, \rho^{\prime}\right)$ is acyclic.

Proof. Suppose there exists a cycle $x^{\prime} \rho^{\prime} y^{\prime} \rho^{\prime} x^{\prime}$ in $\left(P^{\prime}, \rho^{\prime}\right)$. Let $x_{1}, x_{2} \in$ $f^{-1}\left(x^{\prime}\right)$ and $y_{1}, y_{2} \in f^{-1}\left(y^{\prime}\right)$. Since $f$ is a path homomorphism, there exists a path $x_{i} \rho y_{j} \rho x_{k}, i, j, k \in\{1,2\}$ in $(P, \rho)$. If $i=k$, we have an immediate contradiction to the acyclicity of $(P, \rho)$. So we assume $x_{i} \neq x_{k}$. We claim there exists a path $x_{k} \rho x_{i}$ (or $x_{i} \rho x_{k}$ ) so that $x_{k} \rho x_{i} \rho y_{j} \rho x_{k}$ becomes the contradictory cycle. If not, $\left\{x_{i}\right\} \cup\left\{x_{k}\right\}$ constitutes a convex set, but $f\left(\left\{x_{i}\right\} \cup\left\{x_{k}\right\}\right)=\left\{x^{\prime}\right\}$ is not convex. Readily, if $x_{k} \neg \rho x_{i}$, then we use the same argument to assure that $x_{i} \rho x_{k}$ for the same contradiction.

While a path homomorphism guarantees the existence of some path between two pre-image elements such as $x_{i}$ and $x_{k}$, it does not guarantee the existence of the path through any specific element such as $y_{j}$. We might have $x_{i} \rho y_{1}$ and $y_{2} \rho x_{k}$, in which case we us the same argument involving $f\left(\left\{y_{1}\right\} \cup\left\{y_{2}\right\}\right)=y^{\prime}$ where $y^{\prime}$ is not convex will work.

Order preserving functions (homomorphisms) by themselves do not ensure that partial orders (acyclic structures) map onto partial orders. Anti-symmetry may not be preserved. Convex functions, by themselves, also need not preserve the partial order structure, as shown by the example of Figure 4 . The function $g$, as

a

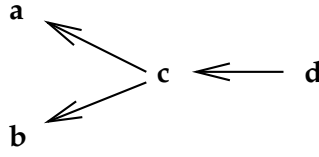

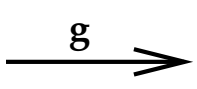

Figure 4. A convex function $g$ that does not preserve the partial order.

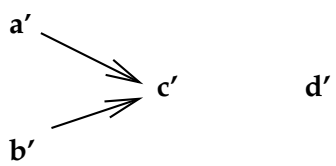

well as any function mapping onto $\left(P^{\prime}, \rho^{\prime}\right)$, must be convex because every subset of $\left(P^{\prime}, \rho^{\prime}\right)$ is convex. Clearly, $g$ is not a homomorphism, and it does not preserve the order of $(P, \rho)$.

It seems impossible to functionally preserve both the structural properties (A2) and (A3) of partial orders with a single functional property, such as order preservation or convexity. 


\section{Closure And Closure lattices}

Closure operators, $\varphi$, which are defined over sets of elements, play a key role in our development. Typically we will consider the closure of subsets $Y \subseteq P$ which we denote by $Y . \varphi$ (read this as $Y$ closure). ${ }^{5}$ Recall that closure operators must satisfy three closure axioms
(a) $Y \subseteq Y . \varphi$
expansive,
(b) $Y \subseteq Z$ implies $Y . \varphi \subseteq Z . \varphi$
monotone, and
(c) $Y . \varphi \cdot \varphi=Y . \varphi$
idempotent.

We call $\left(2^{P}, \varphi\right)$ a closure system. A subset $Y \subseteq P$ is closed if $Y . \varphi=Y$.

There exist many closure operators in the literature, but because we are exploring partial orders we will focus on the three natural path closures, $\varphi_{\downarrow}, \varphi_{\uparrow}$ and $\varphi_{C}$, where for all $Y \subseteq P$,

$$
\begin{aligned}
& Y . \varphi_{\downarrow}=\{x \mid \exists y \in Y, x \rho y\}, \\
& Y . \varphi_{\uparrow}=\{z \mid \exists y \in Y, y \rho z\}, \\
& Y . \varphi_{C}=\left\{y \mid \exists y_{1}, y_{2} \in Y, y_{1} \rho \text { y } \rho y_{2}\right\} .
\end{aligned}
$$

Observe that $\varphi_{\downarrow}$, those elements to the left in our figures, is sometimes called a "downset" operator, or ideal, and that $\varphi_{\uparrow}$, with elements to the right, is called an "upset" operator, or filter, $\varphi_{C}$ has been called a convex closure and its closed subsets called "convex" [27]. A set $Y$ is said to be directed if $x, z \in Y$ implies there exists an upper bound $u b(x, z) \in Y$. If every directed subset, $Y$, has a l.u.b.( $Y)$, the partial order $(P, \leq)$ is called as "directed-complete partial order", or dcpo [1]. Figure 1 is a dcpo with least upper bound $h$.

The closed sets of $\varphi_{C}$ are called "intervals" in [29], or "causal sets" [6,32]. Causal sets are thought to play a role in modeling quantum gravity. When we are modeling temporal events, $Y . \varphi_{\downarrow}$ denotes antecedent events occurring at, or before, the events of $Y, Y . \varphi_{\uparrow}$ denotes descendant events occurring at, or later, than events of $Y$, and $Y . \varphi_{C}$ denotes events occurring "between" events in $Y$ [12].

A closure operator, $\varphi$, that satisfies the anti-exchange axiom,

$$
\forall x \neq z, x, z \notin Y . \varphi \text {, and } z \in(Y \cup\{x\}) . \varphi \text { imply } x \notin(Y \cup\{z\}) . \varphi
$$

is said to be an anti-matroid operator [23]. ${ }^{6}$

Proposition 3.1. If $(P, \rho)$ is a partial order, then $\varphi_{\downarrow}, \varphi_{\uparrow}, \varphi_{C}$ are antimatroid closure operators.

Proof. Let $Y$ be closed with respect to $\varphi_{\downarrow}$ and let $x, z \notin Y$. So $x \neg \rho y, z \neg \rho y, y \in$ $Y$. Now assume $z \in(Y \cup\{x\}) \cdot \varphi_{\downarrow}$, implying that $z \rho x$. Since $(P, \rho)$ is a partial order, $x \neg \rho z$, so $x \notin(Y \cup\{z\}) . \varphi_{\downarrow}$. The proof when $\varphi=\varphi_{\uparrow}$ or $\varphi_{C}$ is similar.

Antimatroid closures are sometimes called "convex geometries" [13,14]. ${ }^{7}$

\footnotetext{
${ }^{5}$ We prefer to use suffix notation when denoting set-valued morphisms such as $\varphi$. It facilitates arrow chasing in categorical diagrams. We reserve the more conventional prefix notation for point functions. The change of notation hopefully alerts the reader to the paradigm shift.

${ }^{6}$ Closure operators that satisfy the "exchange axiom", or "if $x, z \notin Y . \varphi$, but $z \in(Y \cup\{x\}) . \varphi$ then $x \in(Y \cup\{z\}) . \varphi$ ", are called "matroid" closure operators. There is an extensive matroid literature.

${ }^{7}$ Antimatroid closure spaces are far more abundant than one might expect. For example, there exist at least 202 distinct closure spaces comprised of 5 elements. More generally, it can
} 
Let $Y$ be any closed set, by a generator of $Y$, we mean a minimal set $X \subseteq Y$ such that $X . \varphi=Y$. Arbitrary closure operators may have multiple generating sets, but the generating set of an antimatroid closure operator must be unique $[14,30]$. We denote this unique generating set of $Y$ by $Y . \gamma^{8}$ In the literature of convex geometries, elements of the generator are called "extreme points" $[12,14]$.

\subsection{Closure lattices}

Let $\mathcal{C}_{\varphi}$ denote the collection of all subsets of $P$ that are closed with respect to a closure operator, $\varphi$. These may be partially ordered by inclusion to form a complete lower semi-modular lattice in which the greatest element is $P$ (since $P$ must be closed), and least element is, usually, $\emptyset .{ }^{9}$ We call this the closure lattice of $P$ with respect to $\varphi$, and denote it by $\mathcal{L}_{P}$, or $\mathcal{L}_{\varphi}$, depending on the context. Figure 5 shows three different closure lattices arising from the three path closures, $\varphi_{\downarrow}, \varphi_{C}, \varphi_{\uparrow}$, applied to a small 6 element acyclic graph.

\section{$(\mathbf{P}, \rho)$}
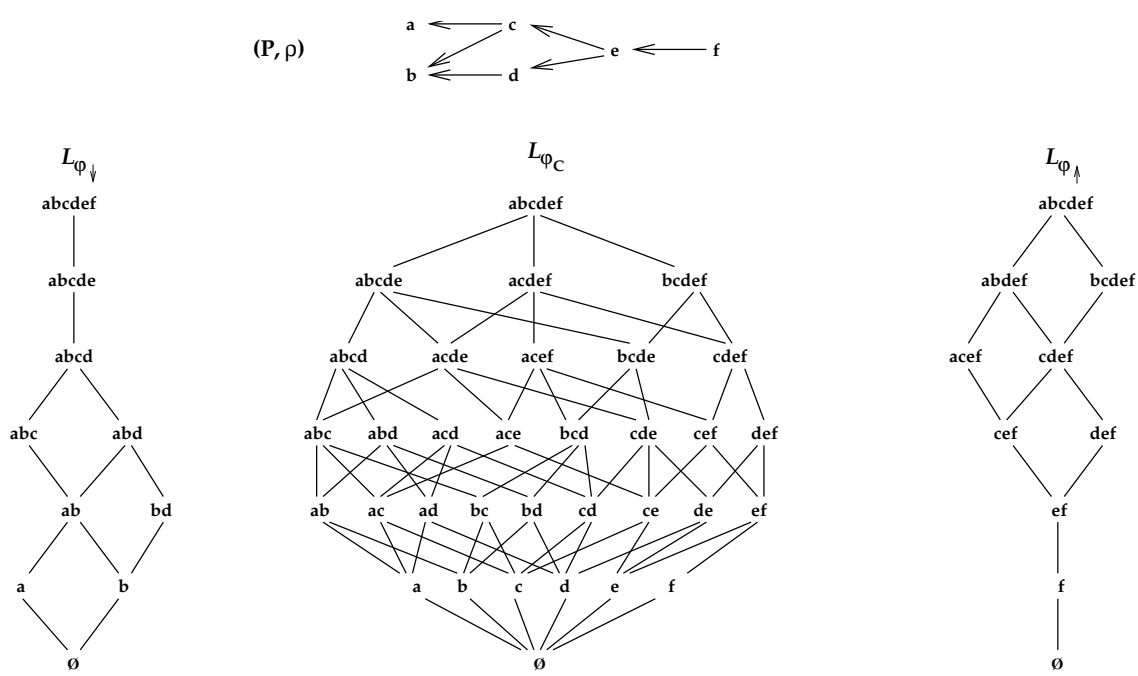

Figure 5. Three closure lattices, $\mathcal{L}_{\downarrow}, \mathcal{L}_{C}, \mathcal{L}_{\uparrow}$.

Proposition 3.2. Let $\varphi$ be an antimatroid closure operator and let $\mathcal{L}_{\varphi}$ be its closure lattice. If $Z$ covers $Y$ in $\mathcal{L}_{\varphi}$ then $Z \backslash Y$ is a singleton.

Proof. Suppose not, that $Z \backslash Y=\{x, y\}, x \neq y$. Then $x \in(Y \cup\{y\}) \cdot \varphi=Z$ and similarly $y \in(Y \cup\{x\}) . \varphi$, contradicting (3.1).

be shown that there exist more than $n^{n}$ distinct, non-isomorphic antimatroid closure spaces provided $n \geq 10$ [28].

${ }^{8}$ So that $\gamma$ is defined for all $Y \subseteq P$, we let $Y \cdot \gamma=Y \cdot \varphi \cdot \gamma$.

${ }^{9}$ It is possible, but unusual, to have a singleton element other than $\emptyset$ be the least element of the lattice. 
So, all covering edges in Figure 5 represent singleton elements. ${ }^{10}$ Moreover, these elements precisely constitute the generating sets of the closed sets above them.

Proposition 3.3. Let $\varphi$ be a path closure with $\mathcal{L}_{\varphi}$ its closure lattice. $Z$ covers $Y_{1}, \ldots Y_{n}$ in $\mathcal{L}_{\varphi}$ if and only if $Z \cdot \gamma=Z \backslash Y_{1} \cup \ldots \cup Z \backslash Y_{n}$.

Proof. Let $\varphi$ be the downset closure $\varphi_{\downarrow}$. Since $\varphi_{\downarrow}$ is antimatroid (Proposition 3.1), $Z \backslash Y_{i}=\left\{z_{i}\right\}$, by Proposition 3.2. Readily, $z_{i}$ is a maximal element of $Z$, since if not, $\exists z \in Z, z_{i} \rho z$ implying $Y_{i}=Z \backslash\left\{z_{i}\right\}$ is not closed. Hence, $z_{i} \in Z . \gamma$.

Conversely, let $z_{i} \in Z . \gamma$ implying $z_{i}$ is maximal in $Z$. Obviously, $\left(Z \backslash\left\{z_{i}\right\}\right) \cdot \varphi_{\downarrow}$ is a closed set which must be covered by $Z$ in $\mathcal{L}_{\varphi_{\downarrow}}$.

The proof for $\varphi_{\uparrow}$ and $\varphi_{C}$ is similar.

Proposition 3.3 is actually true for all antimatroid closures, as shown in [29]. A variant has been established for all closure operators, antimatroid or not, which asserts that $Z$. $\gamma \cap Z \backslash Y_{i} \neq \emptyset$ for all $Y_{i}$ covered by $Z$ in $\mathcal{L} \varphi$, [20]. But, such generality is not needed here.

In $\mathcal{L}_{\varphi_{\downarrow}}$ of Figure 5, abcd covers $a b c$ and $a b d$, with $a b c d \backslash a b c=\{d\}$ and $a b c d \backslash a b d$ $=\{c\}$, so $\{a b c d\} \cdot \gamma=\{c d\}$ which is easily verified in $(P, \rho)$. Similarly in $\mathcal{L}_{C}, a b c d e$ covers $a b c d, a c d e$ and $b c d e$, so $\{a b c d e\} \cdot \gamma=\{e, b, a\}$; the differences between abcde and the closed sets it covers in $\mathcal{L}_{C}$. Finally, bcdef covers cdef and bdef in $\mathcal{L}_{\varphi_{\uparrow}}$. $\{b c d e f\} \cdot \gamma=\{d, c\}$.

In the case of $\mathcal{L}_{\varphi_{\downarrow}}$ and $\mathcal{L}_{\varphi_{\uparrow}}$, given the closure lattice, one can completely reconstruct $(P, \rho)$. It is a direct consequence of the well known fact that a poset $P$ is isomorphic to the set $J D(P)$ of join-irreducible elements of the set of its down-sets. For example, because $\{a b c d\} \cdot \gamma=\{c d\}$ we know $a \rho\{c d\}$ and $b \rho\{c d\}$. But, $\{b d\}$ does not cover $\{a\}$ in $\mathcal{L}_{\varphi_{\downarrow}}$ so $a \neg \rho b$ and $a \neg \rho d$ in $(P, \rho)$. This is not the case for $\mathcal{L}_{C}$. For example, if the set $\{x, y\}$ is closed in $\mathcal{L}_{C}$, we may have $x \rho y$ or $x \neg \rho y$. It is shown in [27], that if there exists an undirected path of length $\geq 2$ in a connected component, the $\mathcal{L}_{C}$ uniquely determines all the undirected paths in that component. Given a single covering relationship (directed edge) in $(P, \rho)$, in a component then all the directed paths in that component are uniquely determined by $\mathcal{L}_{C}$.

We observe in Figure 5 that $\varphi_{C}$ is finer than $\varphi_{\downarrow}$, or $\varphi_{C} \subseteq \varphi_{\downarrow}$, in that $Y \in \mathcal{C}_{\downarrow}$ implies $Y \in \mathcal{C}_{C}$. Similarly, $\varphi_{C} \subseteq \varphi_{\uparrow}$. This follows from the obvious fact that $\varphi_{C}=\varphi_{\downarrow} \cap \varphi_{\uparrow}$.

\section{Closure space transformations}

Sections 2.1 and 2.2 have provided ample evidence of why it is difficult to create a functional "structure preserving" concept that maps discrete partial orders (acyclic graphs) into discrete partial orders based on order preserving point functions over $P$. In this section, we "lift" the discussion to functions on $2^{P}$, the power set over $P$, rather than $P$ itself. In Section 3, we considered closure operators, $\varphi$,

\footnotetext{
${ }^{10}$ Labeling each of the covering edges in $\mathcal{L}_{\varphi}$ can be instructive. It illustrates why Korte, Lovász and Schrader [22] define antimatroid structures in terms of shelling sequences.
} 
which are set-valued functions mapping $2^{P}$ into $2^{P}$. Now we examine functions that map $2^{P}$ into a possibly different power set $2^{P^{\prime}}$, as in [35]. To emphasize the fact that these are functions on $2^{P}$, we will call them transformations, denote them by $\left(2^{P}, \varphi\right) \stackrel{f}{\longrightarrow}\left(2^{P^{\prime}}, \varphi^{\prime}\right)$, and use suffix notation, such as $Y . f$, instead of $f(Y)$. The use of suffix notation when referencing these set-valued operators and functions will help to prevent confusion. However, repeated use of the notation $\left(2^{P}, \varphi\right)$ becomes awkward and clumsy. From now on, we will just write $(P, \varphi)$. Since $2^{P}$ is completely determined by $P$ there is no loss of accuracy.

We assume all transformations $(P, \varphi) \stackrel{f}{\longrightarrow}\left(P^{\prime}, \varphi^{\prime}\right)$ are monotone, that is, $Y \subseteq Z$ implies $Y . f \subseteq Z . f .^{11}$

A transformation $(P, \varphi) \stackrel{f}{\longrightarrow}\left(P^{\prime}, \varphi^{\prime}\right)$ is said to be continuous [10,25, 30,34,35]. if $\forall Y \subseteq P, \quad Y . \varphi . f \subseteq Y . f . \varphi^{\prime}$.

Proposition 4.1. Let $(P, \varphi) \stackrel{f}{\longrightarrow}\left(P^{\prime}, \varphi^{\prime}\right),\left(P^{\prime}, \varphi^{\prime}\right) \stackrel{g}{\longrightarrow}\left(P^{\prime \prime}, \varphi^{\prime \prime}\right)$ be transformations. If both $f$ and $g$ are monotone and continuous, then so is $P \stackrel{f \cdot g}{\longrightarrow} P^{\prime \prime}$.

Proof. We have X. $\varphi . f \subseteq X . f . \varphi^{\prime}$ for any $X \in P$ and $Y . \varphi^{\prime} . g \subseteq Y . g . \varphi^{\prime \prime}$ for any

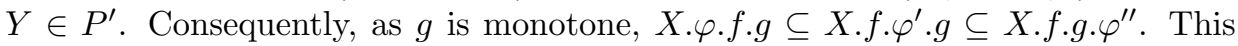
means that $f \cdot g$ is continuous.

That the composition of monotone transformations is monotone is obvious.

A major reason for considering transformations which map $2^{P}$ into $2^{P^{\prime}}$ is that it provides a functional way of adding elements to a partial order, or deleting them. A simple point function $f: P \rightarrow P^{\prime}$ must map every element $y \in P$ to some element $y^{\prime}=f(y) \in P^{\prime}$. A transformation $\left(2^{P}\right) . f$ can map $\{y\} \in 2^{P}$ onto $\emptyset \in 2^{P^{\prime}}$, and thereby delete $\{y\}$ from the partial order. That is, $Y . f=Y \backslash\{y\}$ if $y \in Y$, else it is the identity map. Since we are concerned with continuity and closed sets, transformations can be regarded as lattice morphisms over the respective closure lattices [35]. Figure 6 illustrates the deletion of $d \in P$ of Figure 5 with respect to $\varphi_{C}$. Pre-image equivalence classes are enclosed in dashed lines. They are the closed sets in $2^{P}$ that map onto singleton sets in $2^{P^{\prime}}$. Readily, every set $Y \subseteq P$ is transformed, but in Figures 6 and 7, we use only the lattice of closed sets to depict its behavior.

Figure 7 illustrates the addition of $g^{\prime} \in P^{\prime}$ obtained by letting $Y . f=\left\{Y^{\prime} \cup\left\{g^{\prime}\right\}\right\}$. Here, the dashed lines denote the resulting lattice morphisms induced by $f$. Their relative position in $\mathcal{L}_{\varphi_{\downarrow}}^{\prime}$ determines the paths that must exist in $\left(P, \rho^{\prime}\right)$.

As can be visually tested in figures 6 and 7 , both the transformations are continuous. The use of two distinct closure operators, $\varphi_{C}$ and $\varphi_{\downarrow}$, is deliberate.

\subsection{Extended transformations}

In Section 2, we focused on functions $f: P \rightarrow P^{\prime}$ which preserved essential properties of the partial order $(P, \leq)$, or acyclic graph $(P, \rho)$. A point function $f: P \rightarrow P^{\prime}$

\footnotetext{
${ }^{11}$ Note that monotone transformations preserve set inclusion; isotone functions preserve ordering.
} 
$(\mathbf{P}, \rho)$
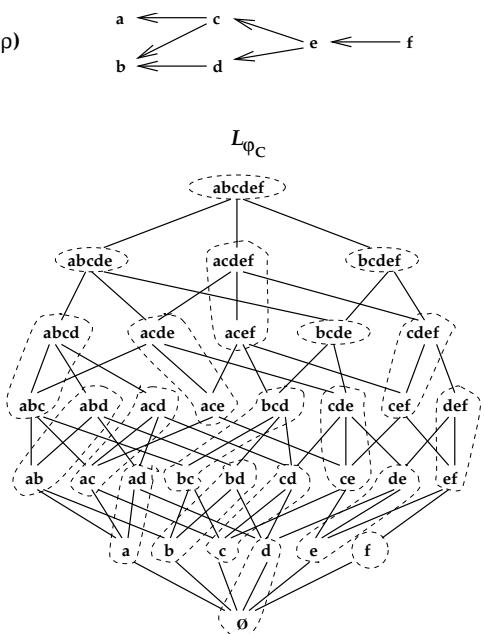
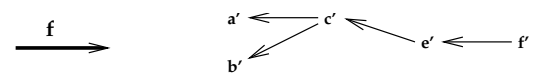

$\left(\mathbf{P}^{\prime}, \rho\right)$

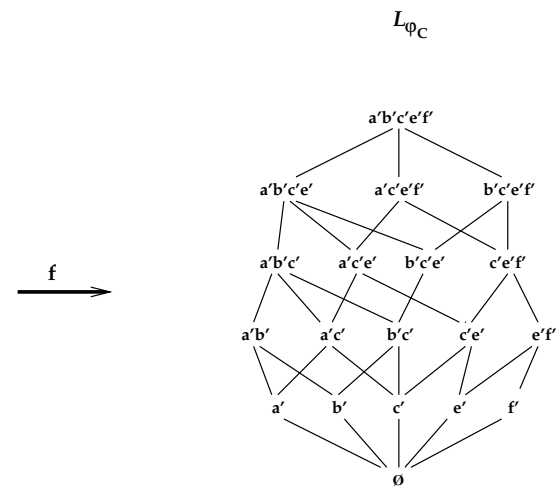

Figure 6. The transformation $f$ deletes $d \in P$ from $P^{\prime}$.

$(\mathbf{P}, \rho)$
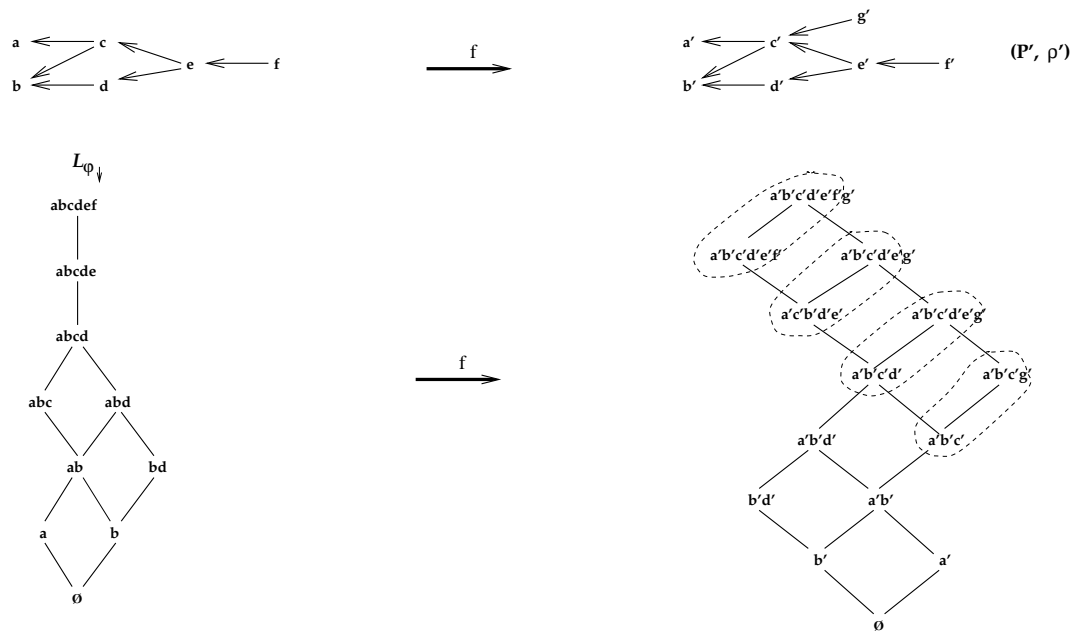

Figure 7. The transformation $f$ adds the new element $g^{\prime}$ to $P^{\prime}$.

can be extended $d^{12}$ to a transformation $2^{P} \stackrel{f^{+}}{\longrightarrow} 2^{P^{\prime}}$ by simply letting

$$
Y \stackrel{f^{+}}{\longrightarrow}=\left\{y^{\prime} \mid \exists y \in Y, y^{\prime}=f(y)\right\}
$$

We use the notation $f^{+}$to denote a transformation that has been so derived. The transformation $f$ of Figure 6 cannot be an extended transformation. The transformation, $g$, of Figure 7 could be an extension of the identity map into $\left(P^{\prime}, \rho^{\prime}\right)$; but

\footnotetext{
${ }^{12}$ This extension of the function $f$, should not be confused with the "linear extension" of the partial order $(P, \rho)$ itself, which is a bijective order preserving function of $(P, \rho)$ onto a total order.
} 
that fails to capture its basic essence. Nevertheless, transformations are frequently defined by extension of a point function, so we examine their properties. Extended transformations must be monotone.

Proposition 4.2. A function $f:(P, \rho) \rightarrow\left(P^{\prime}, \rho^{\prime}\right)$ is path/order preserving if and only if its extended transformation $(P, \varphi) \stackrel{f^{+}}{\longrightarrow}\left(P^{\prime}, \varphi^{\prime}\right)$ is continuous with respect to either the $\varphi_{\downarrow}$ or $\varphi_{\uparrow}$ path closures.

Proof. Let $\varphi$ be the downset closure $\varphi_{\downarrow}$. Let $f$ be path preserving and let $Y \subseteq P . \forall y \in Y$, if $x \rho y$ then $f(x) \rho f(y)$. Consequently, $Y \cdot \varphi_{\downarrow} \cdot f^{+} \subseteq Y . f^{+} \cdot \varphi_{\downarrow}{ }^{\prime}$, so $f^{+}$is continuous.

Conversely, assume $f^{+}$is continuous and that $x \rho y$. Then $x \in\{y\} \cdot \varphi_{\downarrow}$. By monotonicity and continuity, $\{x\} \cdot f^{+} \subseteq\{y\} \cdot \varphi_{\downarrow} \cdot f^{+} \subseteq\{y\} \cdot f^{+} \cdot \varphi_{\downarrow}^{\prime}$ implying $f(x) \rho f(y)$.

The proof when the closure is $\varphi_{\uparrow}$ is similar.

Proposition 4.3. Let $(P, \varphi) \stackrel{f^{+}}{\longrightarrow}\left(P^{\prime}, \varphi^{\prime}\right)$ be an extended transformation. If it continuous with respect to $\varphi_{\downarrow}$ or $\varphi_{\uparrow}$ then it is also continuous with respect to $\varphi_{C}$.

Proof. Suppose $f$ is not continuous w.r.t. $\varphi_{C}$, then $\exists Y \subseteq P$ such that $Y . \varphi_{C} . f \nsubseteq$ $Y . f . \varphi_{C}^{\prime}$ which in turn implies $\exists x \in Y . \varphi_{C}, x \notin Y$ such that $x^{\prime}=x$.f and $x^{\prime} \notin$ $Y . f . \varphi_{C}^{\prime} . x \in Y . \varphi_{C}$ implies $\exists y_{1}, y_{2} \in Y$ and $y_{1} \rho x \rho y_{2}$. But by Proposition 4.2, $f$ continuous w.r.t. $\varphi_{\downarrow}\left(\right.$ or $\left.\varphi_{\uparrow}\right)$ implies $y_{1}^{\prime} \rho^{\prime} x^{\prime} \rho^{\prime} y_{2}^{\prime}$ so $x^{\prime} \in Y . f . \varphi_{C}^{\prime}$, a contradiction.

This is hardly surprising given that $\varphi_{C}=\varphi_{\downarrow} \cap \varphi_{\uparrow}$.

Notably absent from Proposition 4.2 is the convex path closure $\varphi_{C}$, even though by Proposition 4.3, $\varphi_{C}$ must be continuous if either $\varphi_{\downarrow}$ or $\varphi_{\uparrow}$ is. Consider, for example, Figure 4. Readily, $g$ is not order preserving; but $g^{+}$is continuous because every subset of $P$ and $P^{\prime}$ is closed with respect to $\varphi_{C}$.

So "continuous" and "path preserving" are effectively synonyms. But, $f$ need not be a path homomorphism as defined in Section 2.2 in which every path in $P^{\prime}$ has a pre-image path in $P$. Consider the transformation of Figure 7 . The path $b^{\prime} \rho^{\prime} g^{\prime}$ has no pre-image path in $P$. A element $y^{\prime} \in P^{\prime}$ is said to be invertible if $\left\{y^{\prime}\right\} . f^{-1} \neq \emptyset[35]$. The earlier definition of "path homomorphism" in Section 2.2 should be modified to include only invertible elements of $P^{\prime}$.

A transformation $(P, \varphi) \stackrel{f}{\longrightarrow}\left(P^{\prime}, \varphi^{\prime}\right)$ is said to be closed if $Y$ closed in $(P, \varphi)$ implies $Y^{\prime}=Y$. $f$ is closed in $\left(P^{\prime}, \varphi^{\prime}\right)$. This is completely analogous to the topological notion of an "open function" which maps open sets onto open sets.

Proposition 4.4. A transformation $(P, \varphi) \stackrel{f}{\longrightarrow}\left(P^{\prime}, \varphi^{\prime}\right)$ is closed if and only if for all $Y \subseteq P, Y . f . \varphi^{\prime} \subseteq Y . \varphi . f$.

Proof. Let $f$ be closed. $X \subseteq X . \varphi$ implies $X . f \subseteq X . \varphi . f$. But, because $X . \varphi$ is closed and $f$ is closed, $X . f . \varphi^{\prime} \subseteq X . \varphi . f$.

Conversely, let $X$ be closed in $(P, \varphi) . X . f . \varphi^{\prime} \subseteq X . \varphi . f=X . f$. But, readily $X . f \subseteq X . f . \varphi^{\prime}$, so equality holds. 
Proposition 4.5. A transformation $(P, \varphi) \stackrel{f}{\longrightarrow}\left(P^{\prime}, \varphi^{\prime}\right)$ is closed and continuous if and only if for all $Y \subseteq P, Y . \varphi \cdot f=Y . f \cdot \varphi^{\prime}$.

Proof. This follows directly from the definition of "continuity" above and Proposition 4.4 .

Proposition 4.6. A path preserving function $f:(P, \rho) \rightarrow\left(P^{\prime}, \rho^{\prime}\right)$ is a path homomorphism if $(P, \varphi) \stackrel{f^{+}}{\longrightarrow}\left(P^{\prime}, \varphi^{\prime}\right)$ is closed with respect to a path closure.

Proof. Let $x^{\prime} \rho^{\prime} y^{\prime}$ in $P^{\prime}$, and let $Y \in\left\{y^{\prime}\right\} . f^{-1}$. Then $Y . f^{+}=\left\{y^{\prime}\right\}$ so $x^{\prime} \in$ $Y . f^{+} . \varphi_{\downarrow}{ }^{\prime} \subseteq Y \cdot \varphi_{\downarrow}{ }^{\prime} \cdot f^{+}$implying there exists $y_{k} \in Y$ and $x_{i} \in Y . \varphi_{\downarrow}$ such that $x_{i} \rho y_{k}$ where $f\left(x_{i}\right)=x^{\prime}$ and $f\left(y_{k}\right)=y^{\prime}$.

Again, the proof is similar when the closure is $\varphi_{\uparrow}$ or $\varphi_{C}$.

Observe that the converse to this proposition need not be true, as we will demonstrate later with Figure 9. Combining Propositions 4.2 and 4.6, we have

Proposition 4.7. Let $f:(P, \rho) \rightarrow\left(P^{\prime}, \rho^{\prime}\right)$. If $f^{+}$is both closed and continuous with respect to $\varphi_{\uparrow}$ or $\varphi_{\downarrow}$ then $f$ is a path homomorphism.

Proposition 4.8. Let $(P, \rho)$ be a finite partial order, i.e., acyclic directed graph, $\left(P^{\prime}, \rho^{\prime}\right)$ be a directed graph and $f:(P, \rho) \rightarrow\left(P^{\prime}, \rho^{\prime}\right)$ be a surjective point function. If $(P, \varphi) \stackrel{f^{+}}{\longrightarrow}\left(P^{\prime}, \varphi^{\prime}\right)$ is closed with respect to any path closure, then $\left(P^{\prime}, \rho^{\prime}\right)$ is acyclic.

Proof. Assume $\varphi_{\downarrow}$ and that $x^{\prime} \rho y^{\prime}$ and $y^{\prime} \rho x^{\prime}$ constitute a directed cycle in $P^{\prime}$. Let $X=\left\{x^{\prime}\right\} \cdot f^{-1}$ and $Y=\left\{y^{\prime}\right\} \cdot f^{-1}$ be distinct subsets in $P$. Since $f^{+}$is closed with respect to $\varphi$, but $X . f^{+}=\left\{x^{\prime}\right\}$ is not, $X$ cannot be closed. Similarly $Y$ cannot be closed in $P$. By Proposition 4.6, $f$ must be a path homomorphism, so there exists $x_{0} \in X$ and $y_{0} \in Y$ with $y_{0} \in\left\{x_{0}\right\} . \varphi$. Since $\left\{y_{0}\right\} . f^{+}=\left\{y^{\prime}\right\}$ is not closed, there exists $x_{1} \in\left\{y_{0}\right\} . \varphi, x_{1} \in X$. Since $(P, \rho)$ is acyclic $x_{1} \neq x_{0}$.

Next we find $y_{1} \in\left\{x_{1}\right\} \cdot \varphi$, with $y_{1} \neq y_{0}$, and so forth. Since $(P, \rho)$ is finite, this process must terminate, leading to a contradiction.

So having the transformations $(P, \varphi) \stackrel{f^{+}}{\longrightarrow}\left(P^{\prime}, \varphi^{\prime}\right)$ be closed and continuous is sufficient to ensure that $f$ is a path homomorphism and that $\left(P^{\prime}, \rho^{\prime}\right)$ is still a partial order (acyclic). However, these properties are not necessary. In Figure 8, we illustrate an order preserving $f$ whose image is acyclic, but which is not closed with respect to $\varphi_{\downarrow}$. The subset $\{a, b, d\}$ is closed with respect to $\varphi_{\downarrow}$, but its image
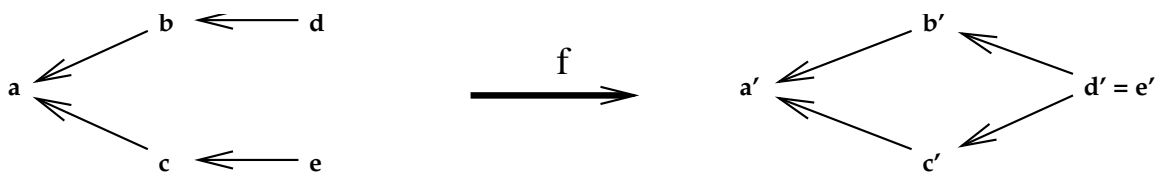

Figure 8. An order preserving/continuous $f$ that is not closed with respect to $\varphi_{\downarrow}$.

$\left\{a^{\prime}, b^{\prime}, d^{\prime}\right\}$ is not. Observe that $f$ is path/order preserving but not a homomorphism by our stronger definition because $\left(c^{\prime}, d^{\prime}\right) \in E^{\prime}$, but there exists no $(c, d) \in E$. 
Proposition 4.8 has in fact proven that if $f^{+}$is continuous and closed then given $x^{\prime} \rho z^{\prime}$ in $P^{\prime}$ then for all $x_{i} \in\left\{x^{\prime}\right\} . f^{-1}$ and all $z_{k} \in\left\{z^{\prime}\right\} . f^{-1}$ there exists a path $x_{i} \rho y_{k}$ in $P$. This is too extreme.

Even path homomorphisms that preserve acyclicity need not be closed, as shown in Figure 9. Would one intuitively regard $g$ as "structure preserving"?
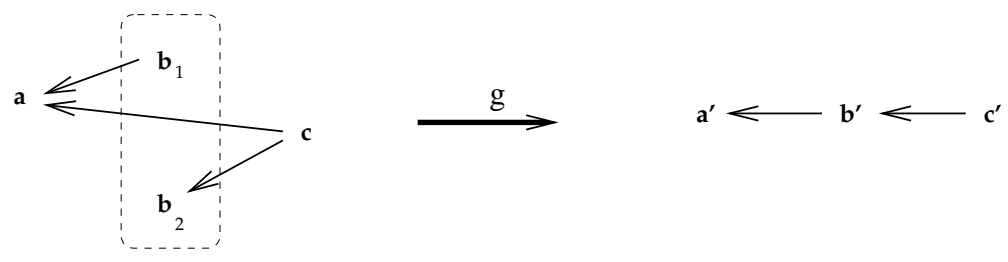

Figure 9. A path homomorphism $g$ that is not closed with respect to $\varphi_{\downarrow}$.

If $Y \subseteq P$ is closed with respect to $\varphi_{C}$ then every path $\left\langle y_{0}, y_{1}, \ldots y_{n}\right\rangle$ with $y_{0}, y_{n} \in Y$ lies completely within $Y$. The transformation $f$ of Figure 8 is closed with respect to $\varphi_{C}$ because only the subsets $\{a d\}$ and $\{a e\}$ are not closed in $P$, and $\left\{a^{\prime} d^{\prime}\right\}$ is not closed in $P^{\prime}$. But, the transformation $g$ of Figure 9 is still not closed with respect to $\varphi_{C}$ because $\{a, c\}$ is closed in $P$, but $\left\{a^{\prime} c^{\prime}\right\}$ is not closed in $P^{\prime}$.

Proposition 4.9. Let $(P, \rho)$ be acyclic and let $f:(P, \rho) \rightarrow\left(P^{\prime}, \rho^{\prime}\right)$ be a path homomorphism. Its image $\left(P^{\prime}, \rho^{\prime}\right)$ is acyclic if and only if $\left(P, \varphi_{C}\right) \stackrel{f^{+}}{\longrightarrow}\left(P^{\prime}, \varphi_{C}^{\prime}\right)$ is continuous and closed.

Proof. "If" follows from Proposition 2.2; we only need show the "only if".

Let $\left(P^{\prime}, \rho^{\prime}\right)$ be acyclic. It is sufficient to show that for any $y^{\prime} \in P^{\prime},\left\{y^{\prime}\right\} . f^{-1}$ is convex in $P$. If not, there exists a path $\left\langle y_{1}, \ldots, y_{n}>\in \rho\right.$ with $y_{1}, y_{n} \in Y$. $f^{-1}$ and an $x \notin Y$.f..$^{-1}$, but $x \in \rho\left(y_{1}, y_{n}\right)$. Now, since $f$ is a path homomorphism, $y^{\prime} \rho^{\prime} x^{\prime}$ and $x^{\prime} \rho^{\prime} y^{\prime}$ contradicting acyclicity.

Proposition 4.9 is stated in terms of extended transformations. We wish a set of criteria that will be applicable to arbitrary transformations $(P, \varphi) \stackrel{f}{\longrightarrow}\left(P^{\prime}, \varphi^{\prime}\right)$.

Let $P \stackrel{f}{\longrightarrow} P^{\prime}$ be a transformation. For all $Y^{\prime} \subseteq P^{\prime}$, by $f^{-1}$ we mean $Y^{\prime} . f^{-1}=$ $\left\{Y \subseteq P \mid Y\right.$.f $\left.=Y^{\prime}\right\}$. So, $f^{-1}$ is a collection of subsets. Let $(P, \rho) \stackrel{f}{\longrightarrow}\left(P^{\prime}, \rho^{\prime}\right)$. As analog to continuity of $f$ we would like to define "inverse continuity" in terms of

$$
Y^{\prime} \cdot \varphi^{\prime} \cdot f^{-1} \subseteq Y^{\prime} \cdot f^{-1} \cdot \varphi
$$

but because $Y^{\prime} \cdot f^{-1}$ is not a subset of $P$, this expression is ill-formed. Applying $f$ to both sides of (4.1) yields

$$
Y^{\prime} \cdot \varphi^{\prime} \subseteq Y^{\prime} \cdot f^{-1} \cdot \varphi \cdot f
$$

and $Y^{\prime} . f^{-1}$ will be replaced by a single element, i.e., a subset of $P$. We say that $f^{-1}$ is inv-continuous with respect to $\varphi$ if there exists a minimal set $Y_{0} \in Y^{\prime} . f^{-1}$ such that

$$
Y^{\prime} \cdot \varphi^{\prime} \subseteq Y_{0} \cdot \varphi \cdot f
$$


A set $Y_{0}$ is minimal if there exists no subset $X \subseteq Y_{0}$ such that $X . f=Y^{\prime}$.

Proposition 4.10. Let $(P, \varphi) \stackrel{f}{\longrightarrow}\left(P^{\prime}, \varphi^{\prime}\right)$ and $\left(P^{\prime}, \varphi^{\prime}\right) \stackrel{g}{\longrightarrow}\left(P^{\prime \prime}, \varphi^{\prime \prime}\right)$ both be inv-continuous, then $(P, \varphi) \stackrel{f . g}{\longrightarrow}\left(P^{\prime \prime}, \varphi^{\prime \prime}\right)$ is inv-continuous.

Proof. Let $Y^{\prime \prime} \subseteq P^{\prime \prime}$. Since $g$ is inv-continuous, $\exists$ a minimal $Y_{0}^{\prime} \subseteq P^{\prime}$ such that $Y^{\prime \prime} \cdot \varphi^{\prime \prime} \subseteq Y_{0}^{\prime} \cdot \varphi^{\prime} . g$. Since $f$ is inv-continuous, $\exists$ a minimal $Y_{0} \subseteq P$ such that $Y_{0}^{\prime} \cdot \varphi^{\prime} \subseteq Y_{0} . \varphi$.f. Consequently, $Y^{\prime \prime} \cdot \varphi^{\prime \prime} \subseteq Y_{0} . \varphi . f . g$. The monotonicity of $\stackrel{f^{+}}{\longrightarrow}$ yields $Y_{0}$ to be a minimal set for $f . g$.

Proposition 4.11. Let $(P, \varphi) \stackrel{f}{\longrightarrow}\left(P^{\prime}, \varphi^{\prime}\right)$ be a transformation and let $(P, \rho)$ be acyclic. If $f^{-1}$ is inv-continuous with respect to $\varphi_{C}$ then $\left(P^{\prime}, \rho^{\prime}\right)$ will be acyclic.

Proof. Let $f$ be inv-continuous w.r.t. $\varphi_{C}$ and suppose $\left(P^{\prime}, \rho^{\prime}\right)$ has a cycle $y^{\prime} \rho^{\prime} z_{1}^{\prime} \ldots z_{k}^{\prime} \rho^{\prime} y^{\prime}$. Let $Y^{\prime}=\left\{y^{\prime}\right\}$ and let $Y=\left\{y_{1}, y_{2}, \ldots y_{n}\right\}=\left\{y^{\prime}\right\} . f^{-1}$. Because $f$ is inv-continuous, w.r.t. $\varphi_{C}$, there exists some $\left\{y_{i}\right\}$ such that $\left\{y^{\prime}, z_{1}^{\prime}, \ldots z_{k}^{\prime}\right\} \subseteq$ $\left\{y_{i}\right\} . \varphi$.f implying that $\left\{y_{i}, z_{1}, \ldots z_{k}\right\}$ constitute a cycle in $(P, \rho)$ which is a contradiction.

The converse need not be true, as shown by Figure 10 .

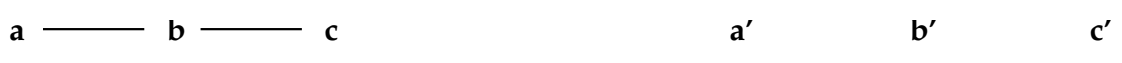

Figure 10. Both $(P, \rho)$ and $\left(P^{\prime}, \rho^{\prime}\right)$ are acyclic but $f$ is not $\varphi_{C}$-inv-continuous.

Using Propositions 4.2 and 4.11 we can assert that if $(P, \rho)$ is a partial order (acyclic), then a transformation $(P, \varphi) \stackrel{f}{\longrightarrow}\left(P^{\prime}, \varphi^{\prime}\right)$ preserves the structure of $(P, \rho)$ if it is continuous with respect to $\varphi_{\downarrow}$, or $\varphi_{\uparrow}$, and inv-continuous with respect $\varphi_{C}$.

\section{Summary}

It is not clear what it means for a mapping $f:(P, \leq) \rightarrow\left(P^{\prime}, \leq^{\prime}\right)$ to be "structure preserving" when $(P, \leq)$ is a partial order. Unless we are prepared to assert that "order preserving" also preserves anti-chains, we cannot claim that every isotone (homomorphic) function $f$ is structure preserving.

In Section 2, we explored conditions on $f^{-1}$ which assure that the image $\left(P^{\prime}, \leq^{\prime}\right)$ would be a partial order. This led to the notion of a convex path homomorphism. (We observe that the concept of a path homomorphism is implicitly dependent on $f^{-1}$.)

In Section 3, we introduced closure concepts and closure lattices, which are themselves partially ordered sets. In an effort to disambiguate the order in the ground structure from that of the associated closure lattice, we used $\rho$ to denote the order in $(P, \leq)$ and $\subseteq$ to denote the order in $\left(2^{P}, \leq\right)$. Every occurrence of $\rho$ and every occurrence of $\subseteq$ can be replace with $\leq$. In some papers this is done. But, we think our notational device, along with suffix notation, makes the mathematics clearer and more intuitive.

While we do not reach a definitive conclusion as to the functional properties of $f$ that characterize it as a "structure preserving" morphism of partial orders, our 
investigations do conclude that such a characterization must be respectful of both (A2), the transitivity of a partial order, and (A3) its anti-symmetry. To this end, it appears that there must be constraints on both the morphism $f$ and its inverse $f^{-1}$.

The equivalent constraints (Proposition 4.2) of path preservation (isotonicity) of $f:(P, \rho) \rightarrow\left(P^{\prime}, \rho^{\prime}\right)$ and continuity (with respect to $\varphi_{\downarrow}$ or $\left.\varphi_{\uparrow}\right)$ of $(P, \varphi) \stackrel{f}{\longrightarrow}\left(P^{\prime}, \varphi^{\prime}\right)$ are sufficient to ensure the expected transitivity. It appears that anti-symmetry requires a convexity constraint on $f^{-1}$, as seen in propositions $2.2,4.9$ and 4.11.

Other constraints on $f$ were considered, such as requiring $(P, \varphi) \stackrel{f}{\longrightarrow}\left(P^{\prime}, \varphi^{\prime}\right)$ to be both continuous and closed (Proposition 4.8); but this was deemed to be far too restrictive, as it requires pre-image paths between every pair of pre-image elements, as illustrated by Figure 8 .

Partially ordered sets are of great importance in mathematical applications, as well as how they morph from one to another. One could argue that, in practice, these are second only to functions $f: R^{m} \rightarrow R^{n}$ which are much more studied. Unfortunately, it feels as if this paper only scratches the surface.

Acknowledgement. The author would like to acknowledge the helpful comments to a previous version of this paper by an unknown referee.

\section{REFERENCES}

[1] S. Abramsky and A. Jung, Domain theory, in: S. Abramsky, D. M. Gabbay and T. S. E. Maibaum (eds.), Handbook of Logic in Computer Science 3, Oxford University Press, 1994, $1-168$.

[2] G. Agnarsson and R. Greenlaw, Graph Theory: Modeling, Applications and Algorithms, Prentice Hall, Upper Saddle River, NJ, 2007.

[3] P. A. Bernstein, V. Hadzilacos and Nathan Goodman, Concurrency Control and Recovery in Database Systems, Addison-Wesley Publ., Reading, MA, 1987.

[4] G. Birkhoff, Lattice Theory, revised ed., American Mathematical Society Colloquium Publications 25, New York, NY, 1948.

[5] G. Birkhoff and M. K. Bennett, The convexity lattice of a poset, Order 2 (1985), 223-242.

[6] G. Brightwell, H. F. Dowker, R. S. Garcia, J. Hensen and R. D. Sorkin, "Observables" in causal set cosmology, Phys. Rev. D 67, (2003), 8 pp.

[7] P. Brinch Hansen, Distributed processes: A concurrent programming concept, Commun. ACM 21 (1978), 934-941.

[8] R. H. Campbell and A. N. Habermann, The specification of process synchronization by path expressions, Lecture Notes in Computer Science 16, Springer, 1974, 89-102.

[9] L. Cardelli, Type systems, in: A. B. Tucker (ed.), The Computer Science and Engineering Handbook, CRC Press, 1997, 2208-2236.

[10] G. Castellini, Categorical Closure Operators, Birkhäuser, Boston, 2003.

[11] G. Chartrand, Graphs as Mathematical Models, Prindle, Weber \& Schmidt, Boston, MA, 1977.

[12] V. Chvátal, Antimatroids, betweenness, convexity, in: W. J. Cook, L. Lovász and J. Vygen (eds.), Research Trends in Combinatorial Optimization, Springer, 2009, 57-64.

[13] W. A. Coppel, Foundations of Convex Geometry, Australian Mathematical Society Lecture Series 12, Cambridge University Press, 1998.

[14] P. H. Edelman and R. E. Jamison, The theory of convex geometries, Geom. Dedicata 19 (1985), 247-270.

[15] B. Ganter and R. Wille, Formal Concept Analysis - Mathematical Foundations, SpringerVerlag, Heidelberg, 1999. 
[16] G. Gierz, K. H. Hoffman, K. Keimel, J. D. Lawson, M. Mislove and D. S. Scott, Continuous Lattices and Domains, Cambridge University Press, 2003.

[17] C. Godsil and G. Royle, Algebraic Graph Theory, Springer, New York, 2001.

[18] G. Gratzer, General Lattice Theory, Academic Press, 1978.

[19] F. Harary, Graph Theory, Addison-Wesley, 1969.

[20] R.E. Jamison and J.L. Pfaltz, Closure spaces that are not uniquely generated, Discrete Appl. Math. 147 (2005), 69-79, also in Ordinal and Symbolic Data Analysis, OSDA 2000, Brussels, Belgium July 2000.

[21] P. T. Johnstone, Stone Spaces, Cambridge Studies in Advanced Mathematics 3, Cambridge University Press, 1982.

[22] B. Korte, L. Lovász and R. Schrader, Greedoids, Springer-Verlag, Berlin, 1991.

[23] S. MacLane, Some interpretations of abstract linear dependence in terms of projective geometry, Amer. J. Math. 58 (1936), 236-240.

[24] S. MacLane, Categories for the Working Mathematician, 2nd ed., Springer-Verlag, New York, 1998.

[25] Ø. Ore, Mappings of closure relations, Ann. Math. 47 (1946), 56-72.

[26] Ø. Ore, Theory of Graphs, American Mathematical Society Colloquium Publications 38, Providence, RI, 1962.

[27] J. L. Pfaltz, Convexity in directed graphs, J. Comb. Theory, Ser. B 10 (1971), 143-162.

[28] J. L. Pfaltz, Evaluating the binary partition function when $N=2^{n}$, Congr. Numer. 109 (1995), 3-12.

[29] J. L. Pfaltz, Closure lattices, Discrete Math. 154 (1996), 217-236.

[30] J. L. Pfaltz and J. Šlapal, Transformations of discrete closure systems, Acta Math. Hungar. 138 (2013), 386-405.

[31] B. C. Pierce, Basic Category Theory for Computer Scientists, MIT Press, Cambridge, MA, 1991.

[32] D. P. Rideout and R. D. Sorkin, Classical sequential growth dynamics for causal sets, Phys. Rev. D 61 (2000), 16 pp.

[33] D. Scott and C. Strachey, Toward a Mathematical Semantics for Computer Languages, Technical Report, Oxford Programming Research Group Technical Monograph, 1971.

[34] J. Šlapal. A Galois correspondence for digital topology, in: K. Denecke, M. Erné and S. L. Wismath (eds.), Galois Connections and Applications, Kluwer Academic, Dordrecht, 2004, 413-424.

[35] J. Šlapal, Complete-lattice morphisms compatible with closure operators, Thai J. Math. 8 (2010), 255-262.

[36] J. Šlapal, On categories of ordered sets with a closure operator, Publ. Math. Debrecen 78 (2011), 61-69.

[37] S. Vickers, Topology via Logic, Cambridge Tracts in Theoretical Computer Science 5, Cambridge University Press, 1989.

John L. Pfaltz, Department of Computer Science, University of Virginia, 85 Engineer's Way, Charlottesville, VA 22904, USA

e-mail: jlp@virginia.edu 